\title{
DISCRETE GROUPS WHOSE MULTIPLIER REPRESENTATIONS ARE TYPE $I$
}

\author{
A. K. HOLZHERR \\ (Received 11 August 1980; revised 22 December 1980) \\ Communicated by G. Brown
}

\begin{abstract}
We determine necessary and sufficient conditions for the multiplier representations of a discrete group to be type $I$. This result extends the corresponding result for ordinary representation given by Kaniuth in [4].
\end{abstract}

1980 Mathematics subject classification (Amer. Math. Soc.): 22 D 10.

\section{Introduction}

Multiplier representations arise in applying the Mackey analysis of ordinary representations of a group $G$ with a regularly embedded normal subgroup $N$. Specifically, to each factor representation of $G$ which restricts to an orbit through $\lambda$ in $N^{*}$, there corresponds a unique $\sigma$-representation of the little group $H_{\lambda} / N$, with the same commutant, where $H_{\lambda}$ is the stabilizer of $\lambda$ in $N^{\wedge}$ and the multiplier $\sigma$ is the Mackey obstruction to extending $\lambda$ to $H_{\lambda}$ as an ordinary representation ([1], page 62). Moreover, if $G$ is discrete, then the induced representation $\lambda \uparrow_{N}^{G}$ has the same commutant as the regular $\sigma$-representation of $H_{\lambda} / N([5]$, Theorem 7).

Suppose, then, that $\omega: G \times G \rightarrow T$ is a normalized multiplier on the discrete group $G$ and let $V(G)$ denote the von Neumann algebra generated by the regular $\omega$-representation of $G$. We say that $(G, \omega)$ is type $I$ if all $\omega$-representations of $G$ are type $I$. The main result to be proved in this paper is the following.

CCopyright Australian Mathematical Society 1981 
THEOREM 1.1. Let $G$ be a discrete group and $\omega$ a normalized multiplier on $G$, then the following are equivalent.

(i) $V(G)$ is type I,

(ii) $(G, \omega)$ is type $I$,

(iii) $G$ has an abelian subgroup $A$ of finite index $[G: A]$ in $G$ such that $\omega(x, y)=\omega(y, x)$ for all $x, y \in A$.

An interesting consequence of this theorem is that the central extension $G^{\omega}$ of $G$ (where $G$ is discrete and $G^{\omega}$ is given any locally compact group topology) is type $I$ if $(G, \omega)$ is type $I$. It should be noted that this is not a simple consequence of the fact that the $\omega$-representations of $G$ give rise to (ordinary) representations of the central extension $G^{\omega}$, since not all representations of $G^{\omega}$ are obtained this way. However, looking at the central extension is a successful approach and we use it in conjunction with Robertson's characterization of Moore groups [7].

In addition to Theorem 1.1, we shall give an explicit description of the maximal type $I$ central projection in $V(G)$ in terms of the properties of $G$, and an application to induced representations.

The results presented in this paper were achieved while the author was working towards a Ph.D. at the University of Adelaide under the supervision of Professor W. Moran. The author is very grateful to Professor Moran for many helpful suggestions.

\section{Notation and some elementary facts}

Throughout this paper we shall be interested in pairs $(G, \omega)$ where $G$ is a discrete group and $\omega$ a normalized multiplier on $G$. For the definitions of multiplier and multiplier representations see Kleppner [5]. A multiplier is normalized if $\omega(x, y)^{-1}=\omega\left(y^{-1}, x^{-1}\right)$ for all $x, y \in G$. Denote by $\rho$ the left regular $\omega^{-1}$-representation of $G$ on $L^{2}(G)$ given by $(\rho(x) f)(y)=\omega\left(x^{-1}, y\right) f\left(x^{-1} y\right)$, $x, y \in G, f \in L^{2}(G)$, and by $V(G)=V(G, \omega)$ the von Neumann algebra generated by $\rho(G)$. If $A(G)=A(G, \omega)$ denotes the twisted group algebra of $(G, \omega)$ the algebra of complex finitely supported measures on $G$ with multiplication $(a b)_{x}=\sum_{y \in G} a_{y} b_{y^{-1} x} \omega\left(y^{-1}, x\right)$-then each $\omega^{-1}$-representation $\pi$ of $G$ extends naturally to an algebra representation $a \rightarrow \Sigma_{g \in G} a_{g} \pi(g)$ of $A(G)$ which, when no confusion may arise, we shall denote by the same letter. With this notation, we see that $V(G)$ is the weak closure of $\rho(A(G))$ in $B\left(L^{2}(G)\right.$ ), the algebra of bounded linear operators on $L^{2}(G)$.

If $\varphi_{x}$ denotes the characteristic function at the point $x$ in $G$, then for $a \in B\left(L^{2}(G)\right), x, y \in G$, we let $a_{x, y}=\left(a\left(\varphi_{y}\right), \varphi_{x}\right)$ and $a_{x}=a_{x, e}$, where $(\cdot, \cdot)$ 
denotes the canonical scalar product on $L^{2}(G)$. Most of the following elementary facts may be found in Kleppner [5]. Let $a \in B\left(L^{2}(G)\right)$, then

2.1. $a \in V(G)$ if, and only if $a_{x, y}=\omega\left(x, y^{-1}\right) a_{x y^{-1}}$ for all $x, y \in G$.

2.2. $a \in C V(G)$, the centre of $V(G)$ if, and only if $\omega(x, y) a_{y-1} x y=$ $a_{x} \omega\left(y, y^{-1} x y\right)$ for all $x, y \in G$.

2.3. For each $a \in V(G)$, the map $g \rightarrow a_{g}$ is in $L^{2}(G)$ and the map $a \rightarrow a_{e}$ is a finite faithful normal trace on $V(G)^{+}$-the set of positive hermitian elements in $V(G)$.

2.4. If $a \in V(G)$, then $a=\Sigma_{g \in G} a_{g} \rho(g)$ in the sense of weak operator convergence. This expression is unique, that is if $a=\sum_{g \in G} a_{g}^{\prime} \rho(g)$, then $a_{g}^{\prime}=a_{g}$ all $g \in G$. We also have

$$
(a b)_{x}=\sum_{y \in G} a_{y} b_{y-1} \omega\left(y^{-1}, x\right) .
$$

2.5. Let $H$ be a subgroup of $G$, then there exists a normal *-isomorphism of $V(H)=V\left(H,\left.\omega\right|_{H \times H}\right)$ onto the von Neumann algebra $\left\{a \in V(G): a_{g}=0\right.$ if $g \notin H\}: a \rightarrow \sum_{h \in H} a_{h} \rho(h), \rho$ being the regular $\omega^{-1}$-representation of $G$.

In view of 2.5 , since we are interested only in the type structure of $V(G)$, we shall henceforth always regard $V(H)$ as a sub von Neumann algebra of $V(G)$ whenever $H$ is a subgroup of $G$.

\section{The $\omega$-finite class group of $G$}

Let $C(x)=C_{G}(x)$ denote the centralizer of $x$ in $G$ and $C_{\omega}(x)=C_{\omega, G}(x)$ the $\omega$-centralizer of $x$ in $G: C_{\omega}(x)=\{g \in C(x): \omega(g, x)=\omega(x, g)\}$.

Lemma 3.1. (i) $C_{\omega}(x)=\{g \in G: \rho(x) \rho(g)=\rho(g) \rho(x)\}$,

(ii) $C_{\omega}(x)$ is a subgroup of $G$.

Proof. If $g \in C_{\omega}(x)$, then

$$
\rho(g) \rho(x) \omega(g, x)=\rho(g x)=\rho(x g)=\rho(x) \rho(g) \omega(x, g)
$$

implies $\rho(g) \rho(x)=\rho(x) \rho(g)$. Conversely, suppose $g \in G$ satisfies $\rho(x) \rho(g)=$ $\rho(g) \rho(x)$, then

$$
\left(\rho(g x) \varphi_{e}\right)(y) / \omega(g, x)=\left(\rho(x g) \varphi_{e}\right)(y) / \omega(x, g)
$$

all $y \in G$, so by letting $y=g x$ and simplifying,

$$
\omega(g, x)^{-1}=\omega\left(g^{-1} x^{-1}, g x\right) \varphi_{e}\left(g^{-1} x^{-1} y\right) / \omega(x, g),
$$

hence $\varphi_{e}\left(g^{-1} x^{-1} y\right)$ cannot be zero, but the only way this can occur is if $x g=y=g x$. Part (i) now follows. The proof of (ii) follows easily from (i). 
Denote by $\Delta=\Delta_{G}$ the $\omega$-finite class group of $(G, \omega)$ defined by $\Delta=\{x \in G$ : [ $\left.\left.G: C_{\omega}(x)\right]<\infty\right\}$. To see that $\Delta$ is indeed a subgroup, we need only note that $C_{\omega}\left(x^{-1} y\right) \supseteq C_{\omega}(x) \cap C_{\omega}(y)$ and

$$
\left[G: C_{\omega}(x) \cap C_{\omega}(y)\right] \leqslant\left[G: C_{\omega}(x)\right]\left[G: C_{\omega}(y)\right] \text {. }
$$

The group $\Delta$ has the property that all its conjugacy classes are finite, such groups are called $F C$ (finite class) groups.

The following theorem points out the significance of $\Delta$.

THEOREM 3.2. Let $G$ be a discrete group, $\omega$ a normalized multiplier and $\Delta$ the $\omega$-finite class group of $G$. If $V(G)$ has a non-zero type I part, then

(i) $[G: \Delta]<\infty$, and

(ii) $\left|\Delta^{\prime}\right|<\infty\left(\Delta^{\prime}\right.$ denotes the commutator subgroup of $\left.\Delta\right)$.

The idea of the proof of (i) comes from Smith ([9], Theorem 9.4) and the proof of (ii) is similar to that of Smith ([10], Theorem 1). We need the following lemma.

Lemma 3.3. Let $C V(G)$ denote the centre of $V(G)$, then $C V(G) \subseteq V(\Delta)$.

Proof. Let $a \in C V(G)$ and $x \in G$ such that $a_{x} \neq 0$, then by 2.5 it suffices to show that $x \in \Delta$. From 2.2, $\omega(x, y) a_{y}{ }^{-1} x y=a_{x} \omega\left(y, y^{-1} x y\right)$, thus $C(x)=C_{\omega}(x)$, but by $2.3,[G: C(x)]<\infty$, hence $x \in \Delta$.

Proof of Theorem 3.2(i). Let $e_{n}$ be a non zero central projection in $V(G)$ such that $e_{n} V(G)$ is type $I_{n}$. From 2.3 we see that $n$ must be finite. Since $e_{n} V(G)$ is a full matrix algebra over its centre $C e_{n} V(G)$, it is of dimemsion at most $n^{2}$ over $C e_{n} V(G) \subseteq e_{n} C V(G) \subseteq C V(G) \subseteq V(\Delta)$. Hence if $g_{1}, \ldots, g_{n^{2}+1}$ are $n^{2}+1$ elements of $G$ belonging to distinct right cosets of $\Delta$, then there exist elements $C_{1}, \ldots, C_{n^{2}+1} \in V(\Delta)$ such that $\sum_{i=1}^{n^{2}+1} C_{i}\left(e_{n} \rho\left(g_{i}\right)\right)=\sum_{i=1}^{n^{2}+1}\left(C_{i} e_{n}\right) \rho\left(g_{i}\right)=0$ with not all $\left(C_{i} e_{n}\right) \rho\left(g_{i}\right)=0$, but $C_{i} e_{n} \in V(\Delta)$, so this cannot happen because the sum $V(\Delta) \rho\left(g_{1}\right) \oplus \cdots \oplus V(\Delta) \rho\left(g_{n^{2}+1}\right)$ is a direct sum of vector spaces. This shows that $[G: \Delta]<n^{2}<\infty$.

LEMMA 3.4. Suppose $G$ is $F C, H$ a subgroup of $G$ and e a projection in $C V(G)$ such that both $\mathrm{eV}(G)$ and $e V(H)$ are type $I_{n}$. If $C=\{x \in G: \rho(h) \rho(x)=$ $\rho(x) \rho(h)$ all $h \in H\}=\cap_{h \in H} C_{\omega, G}(h)$ is the $\omega$-centralizer of $H$ in $G$, then $e(\rho(\sigma) \rho(\tau)-\rho(\tau) \rho(\sigma))=0$ for every $\sigma \in C_{\omega}, \tau \in G$. Consequently $\left[C_{\omega}, G\right]$ (the subgroup generated by elements of the form $\left.\sigma \tau \sigma^{-1} \tau^{-1}, \sigma \in C_{\omega}, \tau \in G\right)$ is finite. 
This result is proved by making appropriate and obvious modifications to Smith [10], Lemma 2.

Proof of Theorem 3.2(ii). It is well known that a von Neumann algebra contains only type $I_{k}$ summands for $k \leqslant n$ if, and only if it satisfies the standard polynomial in $2 n$ variables (for details see for example [11], page 213). Suppose $V(G)$ has a non-zero type $I$ part, then there exists a central projection $e_{n} \neq 0$ such that $e_{n} V(G)$ is type $I_{n}$. By the above remark $e_{n} V(G)$, and hence $e_{n} V(\Delta)$ satisfy the symmetric polynomial of degree $2 n$; consequently $e_{n} V(\Delta)$ is type $I$.

Now let $e_{n}$ be a non-zero central projection in $V(\Delta)$ such that $e_{n} V(\Delta)$ is type $I_{n}$. As in the proof of Smith [10],Theorem 1, we can find a finitely generated normal subgroup $H$ of $\Delta$ such that $e_{n} V(H)$ has a non-zero type $I_{n}$ summand $e V(H)$ for some $e \in C e_{n} V(H)$. We wish to show that $e \in C V(\Delta)$. Because $H$ is normal in $\Delta$, for any $\sigma \in \Delta$, the automorphism $V(H) \rightarrow V(H): a \rightarrow \rho\left(\sigma^{-1}\right) a \rho(\sigma)$ leaves the type $I_{n}$ summand $e V(H)$ fixed, that is $\rho\left(\sigma^{-1}\right) e \rho(\sigma) V(H)=e V(H)$ and so by the uniqueness of $e, \rho\left(\sigma^{-1}\right) e \rho(\sigma)=e$. It follows that $e \in C V(\Delta)$. Since $e<e_{n}, e V(\Delta)$ is clearly also type $I_{n}$, and thus by Lemma $3.4,\left|\left(C_{\omega}\right)^{\prime}\right|<\infty$ where $C_{\omega}=$ $C_{\omega, \Delta}(H)$. Since $H$ is finitely generated, $\left[\Delta: C_{\omega}\right]<\infty$ and thus the result $\Delta^{\prime}<\infty$ follows by [6], Lemma 4.1.

\section{The type I part of $V(G)$}

For any discrete group $G$, we denote by $G_{0}$ the von Neumann kernel of $G$, that is the intersection of the kernels of all finite dimensional (ordinary) representations of $G$. If $H$ is a subgroup of finite index in $G$, then $G_{0}=H_{0}$ (see [3], Satz 7.2.2).

LEMMA 4.1. Let $G$ be a discrete group and $H$ a subgroup of finite index in $G$ such that $\left|H^{\prime}\right|<\infty$, then there exists a subgroup $K$ of $G$ such that $[G: K]<\infty$ and $K^{\prime}=G_{0}=\cap\left\{L^{\prime}:[G: L]<\infty\right\}$.

Proof. If $[G: L]<\infty$ then, since the characters of $L / L^{\prime}$ separate points, we have $G_{0}=L_{0} \subseteq L^{\prime}$, hence $G_{0} \subseteq \cap\left\{L^{\prime}:[G: L]<\infty\right\}$. Since $\left|H^{\prime}\right|<\infty$ and $H / H_{0}$ is maximally almost periodic, by Robertson's characterization of Moore groups ([7], Theorem 1), $H / H_{0}$ is type $I$, so by Kaniuth [4], Satz $2, H$ has a subgroup $K$ containing $H_{0}$, of finite index in $H$ such that $K / H_{0}$ is abelian. Since

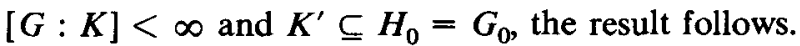


Now let $G$ be a discrete group and $\omega$ a normalized multiplier for $G$. We denote by $G^{\omega}$ the discrete group extension of $T$ by $G$ which is the set $T \times G$ with multiplication $(s, x)(t, y)=(\operatorname{st\omega }(x, y), x y),(s, x),(t, y) \in G^{\omega}$. Note that $G^{\omega}$ is discrete, it is not, as is usual, endowed with the Weil topology. Whenever $H$ is a subgroup of $G$, we identify $H^{\omega}$ in the obvious way with a subgroup of $G^{\omega}$. The following facts are worth noting: (i) $C_{G^{\omega}}(t, x)=\left(C_{\omega}(x)\right)^{\omega}$ for all $(t, x) \in$ $G^{\omega}$, (ii) $\Delta^{\omega}$ is the finite class group of $G^{\omega}$, (iii) $[G: H]=\left[G^{\omega}: H^{\omega}\right]$ for all subgroups $H$ of $G$.

The next lemma is the key step to our proofs of the subsequent results.

LEMMA 4.2. If there exists a subgroup $H$ of $G$ such that $[G: H]<\infty$, $\left|H^{\prime}\right|<\infty$ and $\left.\omega^{n}\right|_{H \times H}$ is trivial for some $n$, then there exists a subgroup $K$ of $G$ such that $[G: K]<\infty, G_{0}=K^{\prime}$ and $\left(G^{\omega}\right)_{0}=\left(K^{\omega}\right)$.

Proof. Because of Lemma 4.1 we can assume without loss of generality that $H^{\prime}=G_{0}$. For some map $\gamma: H \rightarrow T, \omega^{n}(x, y)=\gamma(x) \gamma(y) / \gamma(x y)$, all $x, y \in H$. An easy calculation shows that

$$
\left(H^{\omega}\right)^{\prime}=\left\{\left(\omega(x, y) \omega\left(x^{-1}, y^{-1}\right) \omega\left(x y, x^{-1} y^{-1}\right), x y x^{-1} y^{-1}\right): x, y \in H\right\} .
$$

Since $\left[\omega(x, y) \omega\left(x^{-1}, y^{-1}\right) \omega\left(x y, x^{-1} y^{-1}\right)\right]^{n}=\gamma\left(x y x^{-1} y^{-1}\right)^{-1},\left(H^{\omega}\right)^{\prime}<\infty$. Вy Lemma 4.1, $G^{\omega}$ has a subgroup $M$ such that $\left[G^{\omega}: M\right]<\infty$ and $M^{\prime}=\left(G^{\omega}\right)_{0}$. Let $L$ be the image of the projection $M \rightarrow G:(\lambda, x) \rightarrow x . L$ is a subgroup of $G$ with the property $M \subseteq L^{\omega}$, hence $\left[G^{\omega}: L^{\omega}\right]<\infty$; furthermore $\left(L^{\omega}\right)^{\prime}=M^{\prime}$ and thus $K=L \cap H$ has the desired properties.

THEOREM 4.3. Let $G$ be a discrete group with normalized multiplier $\omega$ and let $e$ (resp. $e_{n}, n=1,2, \ldots, n \neq \infty$ ) be the maximal type $I$ (resp. type $I_{n}$ ) central projections in $V(G)$. By 2.3, $V(G)$ is a finite von Neumann algebra hence $\Sigma_{n<\infty} e_{n}=e$. The following are equivalent.

(a) $e \neq 0$,

(b) there exists a subgroup $H$ of $G$ such that $[G: H]<\infty,\left|H^{\prime}\right|<\infty$ and $\left.\omega\right|_{H \times H}$ is trivial.

Suppose $e \neq 0$, then there exists a 1 dimensional $\omega$-representation $\gamma$ of $G_{0}$ such that

(i) $\left.\pi\right|_{G_{0}}=\operatorname{dim}(\pi) \cdot \gamma$, for all finite dimensional $\omega$-representations $\pi$ of $G$, where $\operatorname{dim}(\pi)$ denotes the dimension of $\pi$, and

(ii) $e=\frac{1}{\left|G_{0}\right|} \Sigma_{g \in G_{0}} \gamma(g) \rho(g)$.

Proof. Suppose $e \neq 0$. Since $e_{n} \neq 0$ for some integer $n$, an irreducible algebra representation $\tau$ of $e_{n} V(G)$ will give rise to a finite dimensional $\omega$-representation 
$\pi: g \rightarrow \tau\left(e_{n} \rho\left(g^{-1}\right)\right)^{*}$ of $G$, where $A^{*}$ denotes the adjoint of $A$ as an operator on the dual space of the Hilbert space on which $A$ acts.

After taking determinants, $\omega^{n}(x, y)$ det $\pi(x y)=\operatorname{det} \pi(x)$ det $\pi(y)$, we see that $\omega^{n}$ is trivial, so by Theorem 3.2 and Lemma 4.2, there exists a group $K$ such that $[G: K]<\infty, G_{0}=K^{\prime}<\infty$ and $\left(G^{\omega}\right)_{0}=\left(K^{\omega}\right)^{\prime}$. Let $\pi$ be an irreducible finite dimensional $\omega$-representation of $K$, then $\pi^{0}:(\lambda, x) \rightarrow \lambda \pi(x)$ is a finite dimensional representation of $K^{\omega}$, so

$$
\pi^{0}\left((1, x)(1, y)(1, x)^{-1}(1, y)^{-1}\right)=\pi(x) \pi(y) \pi(x)^{-1} \pi(y)^{-1}=I \quad \text { all } x, y \in K,
$$

but $\pi$ is irreducible so it is one dimensional, consequently $\left.\omega\right|_{K \times K}$ is trivial. This proves (a) implies (b).

Conversely, suppose (b) is true. Since $\left.\omega\right|_{H \times H}$ is trivial, $H$ has a 1 dimensional $\omega$-representation, hence by inducing we see that $G$ possesses a finite dimensional $\omega$-representation. Let $\pi$ be such a representation. Lemma 4.2 is applicable, so as in the preceding paragraph, $\pi(x) \pi(y)=\pi(y) \pi(x)$ all $x, y \in K$, and $G_{0}=K^{\prime}$, where $K$ is the subgroup we obtain from Lemma 4.2 , consequently

$$
\pi\left(x y x^{-1} y^{-1}\right)=\omega(y, x) \omega\left(y^{-1}, x^{-1}\right) \omega\left(y x, y^{-1} x^{-1}\right) . I .
$$

Since the left hand side of this expression is independent of the way we express $x y x^{-1} y^{-1}$ as a commutator, and since the right hand side depends only on the dimension of $\pi$, the function

$$
\gamma: G_{0} \rightarrow T: x y x^{-1} y^{-1} \rightarrow \omega(y, x) \omega\left(y^{-1}, x^{-1}\right) \omega\left(y x, y^{-1} x^{-1}\right), \quad x, y \in K,
$$

extends to a well defined $\omega$-representation of $G_{0}$ that satisfies (i).

To complete the proof of this theorem, we must show that our current assumptions lead to the statements (a) and (ii). Let $f=\left|G_{0}\right|^{-1} \Sigma_{g \in G_{0}} \gamma(g) \rho(g)$. By 2.2, $f$ is central in $V(G)$ and since $g \rightarrow \gamma(g) \rho(g)$ is an ordinary representation of $G_{0}, f^{2}=f$. Now, using the relation $\pi(x) \pi(y)=\pi(y) \pi(x)$ all $x, y \in K$, and the fact that $G_{0}=K^{\prime}$, one can show that $f V(K)$ is abelian. If we write $f V(G)$ as the module direct sum $f V(K) \rho\left(g_{1}\right) \oplus \cdots \oplus f V(K) \rho\left(g_{k}\right)$ for some set of coset representatives $g_{1}, \ldots, g_{k}$ modulo $K$, then (as in Smith [10], page 404) $f V(G)$ is a matrix algebra over the abelian algebra $f V(K)$ and is thus type $I$ ([11], page 213). This proves (a).

By looking at the irreducible algebra representations of $e_{n} V(G)$, whenever this is not zero, we see that $e V(G)$ has enough finite dimensional representations to separate the points of $e V(G)$. But if $\pi$ is a non-zero finite dimensional representation of $e V(G), g \rightarrow \pi\left(\rho\left(g^{-1}\right)\right)^{*}$ is a finite dimensional $\omega$-representation of $G$, hence by (i), $\pi(\rho(g) \gamma(g))=1$ all $g \in G_{0}$, hence $\pi(f)=\left|G_{0}\right|^{-1} \Sigma_{g \in G_{0}} \pi(\rho(g) \gamma(g))$ $=I$, from which we obtain $\pi(e-f)=\pi(e)-\pi(f)=0$, and since $\pi$ was arbitrary, we conclude that $e=f$. This completes the proof of Theorem 4.3. 
Note that for $\omega$ trivial this theorem reduces to results due to Formanek [2] and Schlichting [8]. As a corollary, we can now prove Theorem 1.1.

Proof of Theorem 1.1. Suppose (i) is true, then by the proof of Theorem 4.3, there exists a group $K$ such that $[G: K]<\infty, K^{\prime}=G_{0}$ and $\left.\omega\right|_{K \times K}$ trivial. Since the maximal type $I$ central projection of $V(G)$ is the identity, by Theorem 4.3, $I=\left|G_{0}\right|^{-1} \Sigma_{g \in G_{0}} \gamma(g) \rho(g)$, hence by uniqueness (see 2.4), $G_{0}=\{e\}$ and thus $K$ is abelian. From $\left.\omega\right|_{K \times K}$ trivial, we can now conclude that $\omega(x, y)=\omega(y, x)$ all $x, y \in K$. This proves (i) implies (iii).

Suppose (iii) is true. Let $H$ be a subgroup as prescribed in (iii) and $\pi$ an $\omega^{-1}$-representation of $G$. If $g_{1}, \ldots, g_{n}$ is a set of coset representatives modulo $H$, then

$$
A(G)=A(H) g_{1} \oplus \cdots \oplus A(H) g_{n}
$$

where the direct sum is a module direct sum (for a definition of $A(G)$ see Section 2). Hence $A(G)$ is a matrix algebra over the abelian algebra $A(H)$ (see Smith [10], page 404) and thus satisfies a polynomial identity. Since the von Neumann algebra generated by $\pi$ is the weak closure of $\pi(A(G))$, it too satisfies a polynomial identity and thus (see [11], page 213) the von Neumann algebra generated by $\pi$ is type $I$. This proves (iii) implies (ii).

The implication (ii) implies (i) is trivial.

By doing some extra calculations, one can prove that the abelian subgroup in Theorem 1.1 may be taken to be $Z_{\omega}(\Delta)=\{g \in Z(\Delta): \omega(g, x)=\omega(x, g)$ all $x \in \Delta\}$, where $Z(\Delta)$ denotes the centre of $\Delta$.

Keeping this in mind, we have the following examples.

EXAMPLE 4.4. Let $G$ be abelian and discrete with normalized multiplier $\omega$.

If $Z_{\omega}(G)=\{g \in G: \omega(x, g)=\omega(g, x)$ all $x \in G\}$ has finite index in $G$, then clearly $(G, \omega)$ is type $I$. Conversely, if $(G, \omega)$ is type $I$, then for each $x \in G$, $C_{\omega}(x)$ contains the group $Z_{\omega}(\Delta)$ which according to Theorem 1.1 , has finite index in $G$, thus $\Delta=G$ and $\left[G: Z_{\omega}(G)\right]<\infty$.

This criterion is well known and applies to pairs such as $G=Z \times Z$, $\omega\left((m, n),\left(m^{\prime}, n^{\prime}\right)\right)=e^{2 \pi i \alpha\left(m n^{\prime}-m^{\prime} n\right)}$ for which $(G, \omega)$ is type $I$ if, and only if $\alpha$ is rational.

EXAMPLE 4.5. Let $p$ be a prime and let $G$ be the group with infinitely many generators $b, a_{1}, a_{2}, \ldots$ and the defining relations

$$
\begin{aligned}
b^{p} & =a_{1}^{p}=a_{2}^{p}=\ldots=a_{n}^{p}=\ldots=1, \\
a_{i} b & =b a_{i}, \quad i=1,2, \ldots, \\
a_{i+k} a_{i} & =b a_{i} a_{i+k}, \quad i, k=1,2, \ldots
\end{aligned}
$$


It is clear that the commutator subgroup of $G$ coincides with the centre of $G$ and is equal to the finite cyclic group $\langle b\rangle$. This implies that there is a bound on the size of the conjugacy classes of $G$.

Let $\omega$ be the multiplier $\omega\left(b^{s_{0}} a_{1}^{s_{1}} a_{2}^{s_{2}} \ldots a_{n}^{s_{n}}, b^{s_{0}^{\prime}} a_{1}^{s_{1}^{\prime}} a_{2}^{s_{2}^{\prime}} \ldots a_{n}^{s_{n}^{\prime}}\right)=e^{2 \pi i / P\left(s_{1} s_{2}-s_{1} s_{2}\right)}$. The elements of the form $b^{s_{0}} a_{3}^{s_{3}} a_{4}^{s_{4}} \ldots a_{n}^{s_{n}}$ form a normal subgroup $H$ of $G$ that is contained in $\Delta$, hence $[G: \Delta]<\infty$; also $\left|\Delta^{\prime}\right| \leqslant\left|G^{\prime}\right|=p$, so by Theorem 4.3, $V(G, \omega)$ has a nonzero maximal type $I$ part. However, because $Z(\Delta) \cap H \subseteq$ $Z(H)=\langle b\rangle$, the group $Z(\Delta)$ and consequently $Z_{\omega}(\Delta)$ has finite index in $G$, thus by Theorem 1.1, V(G, $\omega)$ is not type $I$.

\section{Induced characters}

The aim of this section is to prove the following theorem.

THEOREM 5.1. Let $G$ be a discrete group and $N$ a normal subgroup of $G$. If $\lambda$ is a character of $N$, that is a 1 dimensional representation of $N$, denote by $H_{\lambda}=\{g \in$ $G:$ gng $^{-1} n^{-1} \in \operatorname{ker} \lambda$ all $\left.n \in N\right\}$ the stabilizer of $\lambda$, then the von Neumann algebra $V_{\lambda}$ generated by the induced representation $\lambda \uparrow_{N}^{G}$ is type I if, and only if $H_{\lambda}$ contains a subgroup $A$ such that $\left[H_{\lambda}: A\right]<\infty$ and $A^{\prime} \subseteq \operatorname{ker} \lambda$.

LEMMA 5.2. (Kleppner [5], Theorem 7). Assume the notation of the above theorem. If $\omega$ is a normalized multiplier of $H_{\lambda} / N$ obtained from an extension of $\lambda$ to $H_{\lambda}\left([5]\right.$, Theorem 5), then there exists a normal ${ }^{*}$-isomorphism between $V_{\lambda}$ and $V\left(H_{\lambda} / N\right)=V\left(H_{\lambda} / N, \omega\right)$.

Proof of THeorem 5.1. Let $P \subseteq H_{\lambda}$ be a set of coset representatives modulo $N$ (including the identity of $H_{\lambda}$ ). For each $x \in H_{\lambda}$ denote by $\alpha_{x}$ the unique element in $P$ such that $x N=\alpha_{x} N$. Let $\psi$ be the function $G \rightarrow N: x \rightarrow \alpha_{x}^{-1} x$. The relations $\psi(n)=n, \psi(x n)=\psi(x) n$ and $\psi(n x)=\psi(x) x^{-1} n x$, for $x \in H, n \in N$ are evident. It is clear that $\tilde{\lambda}: x \rightarrow \lambda(\psi(x))$ is an extension of $\lambda$ to $H_{\lambda}$ and that the multiplier $\sigma(x, y)=\lambda(\psi(x y)) \lambda(\psi(x) \psi(y))^{-1}$ associated with this extension can be factored through $H_{\lambda} / N$ to yield a multiplier $\omega$ on $H_{\lambda} / N$. We may assume without loss of generality that $\omega$ is normalized. Using Theorem 1.1 and Lemma 5.2, we deduce that $V_{\lambda}$ is type $I$ if, and only if there exists a subgroup $A$ in $H_{\lambda}$ such that both (i) $\left[H_{\lambda}: A\right]<\infty$ and (ii) $A^{\prime} \subseteq N, \sigma(x, y)=\sigma(y, x)$ all $x, y \in A$, hold. It remains to show that (ii) is equivalent to $A^{\prime} \subseteq \operatorname{ker} \lambda$. Suppose (ii) is true. Let $x, y \in A$. Since $x^{-1} y^{-1} x y \in N, \psi(x y)=\psi\left(y x x^{-1} y^{-1} x y\right)=\psi(y x) x^{-1} y^{-1} x y$, but from $\sigma(x, y)=\sigma(y, x)$ follows $\lambda(\psi(y x))=\lambda(\psi(x y))$ so that $\lambda\left(x^{-1} y^{-1} x y\right)=$ 1. Conversely if $A^{\prime} \subseteq$ ker $\lambda \subseteq N$, then for $x, y \in A, \lambda(x y)=\lambda\left(\psi\left(y x x^{-1} y^{-} x y\right)\right)=$ $\lambda(\psi(y x)) \lambda\left(x^{-1} y^{-1} x y\right)=\lambda(\psi(y x))$ and thus $\sigma(x, y)=\sigma(y, x)$. This completes the proof. 


\section{Concluding remarks}

Given a discrete group $G$ with a normalized multiplier $\omega$, Theorem 3.2 serves the purpose of obtaining a subgroup of finite index in $G$ whose commutator subgroup is finite, provided $V(G, \omega)$ has a non-zero type $I$ part. Such a subgroup can also be obtained by combining Kleppner ([5], page 564) with Taylor's version ([11], Theorem 3) of Kanuith's result ([12], Satz 2).

As a consequence of Theorem 1.1, one finds that for locally compact groups $G$ with normalized Borel multiplier $\omega$, Theorem 1.1 can be generalized along the lines of Moore ([13], Theorem 1) and Taylor ([11], Theorem 2).

\section{References}

[1] L. Auslander and C. C. Moore, 'Unitary representations of solvable Lie groups', Mem. Amer. Math. Soc. 62 (1966).

[2] E. Formanek, 'The type I part of the regular representation', Canad. J. Math. 26 (1974), 1086-1089.

[3] H. Heyer, Dualität lokalkompakter Gruppen (Lecture Notes in Math. 150, Berlin, 1970).

[4] E. Kaniuth, 'Der Typ der regulären Darstellung diskreter Gruppen', Math. Ann. 182 (1969), $334-339$.

[5] A. Kleppner, 'The structure of some induced representations', Duke Math. J. 29 (1962), 555-572.

[6] B. H. Neumann, 'Groups with finite classes of conjugate subgroups', Math. Z. 63 (1955), 76-96.

[7] L. C. Robertson, 'A note on the structure of Moore groups', Bull. Amer. Math. Soc. 75 (1969), 594-599.

[8] G. Schlichting, 'Eine Charakterisierung gewisser diskreter Gruppen durch ihre reguläre Darstellung', Manuscripta Math. 9 (1973), 389-409.

[9] M. Smith, 'Group algebras', J. Algebra 18 (1971), 477-499.

[10] _ 'Regular representations of discrete groups', J. Functional Analysis 11 (1972), 401-406.

[11] K. F. Taylor, 'The type structure of the regular representation of a locally compact group', Math. Ann. 222 (1976), 211-224.

[12] E. Kaniuth, 'Die Struktur der regulären Darstellung lokalkompakter Gruppen mit invarianter Umgebungsbasis der Eins', Math. Ann. 194 (1971), 225-248.

Department of Mathematics

The University of Adelaide

Adelaide, S.A. 5001

Australia 